\title{
Multiband emission from pulsar wind nebulae: a possible injection spectrum
}

\author{
J. Fang and L. Zhang
}

Department of Physics, Yunnan University, Kunming, PR China

e-mail: lizhang@ynu.edu.cn

Received 6 November 2009 / Accepted 11 February 2010

\section{ABSTRACT}

\begin{abstract}
Aims. Recent research shows that particles with a spectrum of a relativistic Maxwellian plus a high-energy tail can be accelerated by relativistic collisionless shocks. We investigate the possibility of the high-energy particles with this new spectrum injected into pulsar wind nebulae (PWNe) from the terminate shock based on the study of multiwavelength emission from PWNe.

Methods. The dynamics of a supernova remnant (SNR) and multiband nonthermal emission from the PWN inside the remnant are investigated using a dynamical model with electrons/positrons injected with the new spectrum. In this model, the dynamical and radiative evolution of a pulsar wind nebula in a nonradiative supernova remnant can be described self-consistently.

Results. This model is applied to the three composite SNRs, G0.9+0.1, MSH 15-52, G338.3-0.0, and the multiband observed emission from the three PWNe is reproduced.

Conclusions. Our studies on the three remnants provide evidence of a new spectrum of the particles, which are accelerated by the terminate shock and injected into a PWN.
\end{abstract}

Key words. gamma rays: ISM - ISM: individual objects: G0.9+0.1 - ISM: individual objects: MSH 15-52 -

ISM: individual objects: G338.3-0.0 - acceleration of praticles - radiation mechanisms: non-thermal

\section{Introduction}

PWNe are prominent sites of high-energy emission in the Galaxy that are powered by the pulsars associated with them. A pulsar inside a PWN loses its rotational energy through a pulsar wind composed of magnetic flux and high-energy particles (Goldreich \& Julian 1969; Gelfand et al. 2007, 2009). The ultrarelativistic wind flows relativistically into a nonrelativistic ejecta of the ambient supernova remnant (SNR), which results in the PWN and a termination shock (TS), where the plasma is decelerated and heated (Reynolds \& Chevalier 1984; Volpi et al. 2008). High-energy particles are injected into the nebula from the TS, and multiband nonthermal photons with energies ranging from radio, $\mathrm{X}$-ray, to $\gamma$-ray bands are emitted during the evolution of the PWN

Usually, multiwavelength observational results of a PWN cannot be reproduced well by the radiation of particles injected with a single power-law spectrum, and a broken power-law must be employed to explain the observations better (e.g., Atoyan \& Aharonian 1996; Aharonian et al. 2005a; Zhang et al. 2008). However, the physics behind the broken power law is unclear. For a typical PWN, the wind from the pulsar can flow relativistically with a Lorentz factor of $\sim 10^{6}$. The resulting TS can accelerate particles to relativistic energy. On the other hand, based on the long-term, two-dimensional particle-in-cell simulations, Spitkovsky (2008) finds that the particle spectrum downstream of a relativistic shock consists of two components: a relativistic Maxwellian and a high-energy power-law tail with an index of $-2.4 \pm 0.1$. Based on this finding and with the assumption that the high-energy particles in a PWN are injected with a spectrum of a relativistic Maxellian plus a power-law high-energy tail, we investigate the multiwavelength emission from PWNe to test the possibility of particles with the new spectrum injected into the PWNe.

The dynamical evolution of the PWN is calculated basically according to the model in Gelfand et al. (2009), which can self-consistently describe the dynamical and radiative evolution of a pulsar wind nebula in a nonradiative supernova remnant. Different from Gelfand et al. (2009), in which a single powerlaw injection spectrum for the electrons/positrons is employed to discuss the radiative properties during different phase of the PWN, we argue in this paper that the high-energy particles are injected with the new spectrum of a relativistic Maxellian plus a power-law, high-energy tail during the evolution in this paper, and a kinetic equation is used to obtain the energy distribution of the particles. We apply the model to the PWNe in the composite SNRs, G0.9+0.1, MSH 15-52, and G338.3-0.0, which have been observed in radio, X-rays and very high-energy (VHE) $\gamma$-rays.

G0.9+0.1 has a $2^{\prime}$ PWN inside a $8^{\prime}$ shell in the radio band (Helfand \& Becker 1987). The PWN is powered by an energetic pulsar PSR J1747-2809, which has recently been discovered in G0.9+0.1 with the NRAO Green Bank Telescope at $2 \mathrm{GHz}$ (Camilo et al. 2009). A jet-like feature of the nebula was revealed in the high-resolution observation with Chandra (Gaensler et al. 2001). The observation in the TeV band with HESS indicates that the PWN is a weak emitter in VHE $\gamma$-rays (Aharonian et al. 2005a).

MSH 15-52 is a complex SNR with a ragged shell in the radio observations (Caswell et al. 1981). An elongated PWN powered by an energetic pulsar was found in the remnant in the X-ray observations with ROSAT (Trussoni et al. 1996), BeppoSAX (Mineo et al. 2001) and Chandra (Gaensler et al. 2002). The remnant has also been well detected in VHE $\gamma$-rays with HESS 
(Aharonian et al. 2005b) and CANGAROO (Nakamori et al. 2008), and the significant VHE $\gamma$-rays are identified as produced by the PWN.

A VHE $\gamma$-ray source HESS J1640-465 was discovered by HESS in the survey of the inner Galaxy (Aharonian et al. 2006), and it is spatially coincident with the composite SNR G338.30.0 . An extended X-ray source was found to be located at the center of the VHE $\gamma$-ray source with XMM-Newton (Funk et al. 2007). Lemiere et al. (2009) presents the high resolution X-ray observations on the PWN, and a point-like source as a putative pulsar appears in the X-ray observations.

In this paper, we investigate the possibility of particles with the new spectrum injected in PWNe based on applications with the spectrum to the three composite SNRs. In Sect. 2, the model is simply described, and the results from the applications to the three SNRs G0.9+0.1, MSH 15-52, and G338.3-0.0, are presented. The main conclusions and discussion are given in Sect. 3.

\section{Model and results}

A PWN is powered by the its pulsar which dissipates the rotational energy into the nebula. The spin-down luminosity of a neutron star with a rotation period of $P$ evolves with time as (e.g., Gaensler \& Slane 2006; Slane 2008)

$\dot{E}(t)=\dot{E}_{0}\left(1+t / \tau_{0}\right)^{-(n+1) /(n-1)}$,

where $\tau_{0}$ is the spin-down time scale of the star, $\dot{E}_{0}$ the initial spin-down power, $n$ the braking index of the pulsar, which is equal to 3 for magnetic dipole spindown.

Magnetic field and high-energy particles are injected at the TS located where the ram pressure of the unshocked wind is equal to that of the PWN. In this paper, we assume the spin-down power is distributed between electrons and positions $\left(\dot{E}_{\mathrm{e}}(t)=\right.$ $\left.\eta_{\mathrm{e}} \dot{E}(t)\right)$ and magnetic fields $\left(\dot{E}_{\mathrm{B}}=\eta_{\mathrm{B}} \dot{E}(t)\right)$ (e.g., Gelfand et al. 2009). Gelfand et al. (2009), use a single power-law injection spectrum for the electrons/positrons to discuss the radiative properties during different phases of the PWN evolution. However, a broken power-law spectrum is usually needed to reproduce the nonthermal emission of a PWN with multiband observations (e.g., Atoyan \& Aharonian 1996; Venter \& de Jager 2006; Slane et al. 2008; Zhang et al. 2008). Recently, based on the long-term, two-dimensional particle-in-cell simulations, Spitkovsky (2008) has found that the particle spectrum downstream of a relativistic shock can be fitted as a Maxwellian plus a power-law tail. The form is $f(\gamma)=C_{1} \gamma \exp \left(-\gamma / \Delta \gamma_{1}\right)+$ $C_{2} \gamma^{-\alpha} \min \left[1, \exp \left(-\gamma-\gamma_{\text {cut }}\right) / \Delta \gamma_{\text {cut }}\right]$, where $\gamma$ is the Lorentz factor of the particles, $\Delta \gamma_{1}$ is close to $\left(\gamma_{0}-1\right) / 2, \gamma_{0}$ is the Lorentz factor of the upstream flow, $C_{2}=0$ for $\gamma<\gamma_{\min }, \gamma_{\min }$ is about seven times of $\Delta \gamma_{1}$ (Spitkovsky 2008). In this paper, we investigate the possibility of particles with this spectrum injected in PWNe by multiwavelength studies.

We argue that high-energy particles injected in a PWN are accelerated by the TS, for which the upstream pulsar wind typically can have a Lorentz factor $\sim 10^{6}$. Therefore, we assume the spectrum of the high-energy particles injected in the PWN has the form

$Q(E, t)=$

$$
C(t) \begin{cases}\frac{E}{E_{\mathrm{b}}} \mathrm{e}^{-\frac{E}{E_{\mathrm{b}}}} & E \leq E_{\min } \\ {\left[\frac{E}{E_{\mathrm{b}}} \mathrm{e}^{-\frac{E}{E_{\mathrm{b}}}}+f\left(\frac{E}{E_{\mathrm{b}}}\right)^{-\alpha}\right]} & E_{\min }<E \leq E_{\max },\end{cases}
$$

where, $\alpha=2.4 \pm 0.1, E_{\mathrm{b}} \sim 2.6 \times 10^{5} \gamma_{\mathrm{ts}, 6} \mathrm{MeV}, \gamma_{\mathrm{ts}, 6}$ is the Lorentz factor of the upstream pulsar wind of the TS in units of $10^{6}, E_{\min }=f_{\min } E_{\mathrm{b}}$ with $f_{\min } \sim 7$, and $f$ is normalized by $E_{\min } / E_{\mathrm{b}} \exp \left(-E_{\min } / E_{\mathrm{b}}\right)=f\left(E_{\min } / E_{\mathrm{b}}\right)^{-\alpha}$. This spectrum is similar to the one given in Spitkovsky (2008) except for a discontinuity at $E_{\min }$, which has no influence on the final multiband photon spectrum, and the cut-off form at the highest energy. We leave $E_{\max }$ as a parameter constrained by the observational results for a PWN. $C(t)$ can be obtained with

$$
C(t)=\frac{\dot{E}_{\mathrm{e}}(t)}{2 E_{\mathrm{b}}^{2}+f \frac{E_{\mathrm{b}}^{2}}{2-\alpha}\left[\left(\frac{E_{\max }}{E_{\mathrm{b}}}\right)^{2-\alpha}-\left(\frac{E_{\min }}{E_{\mathrm{b}}}\right)^{2-\alpha}\right]} .
$$

Assuming the particles are homogeneously distributed in space in the PWN, and the distribution in the emission region is given by

$\frac{\partial N(E, t)}{\partial t}=\frac{\partial}{\partial E}[\dot{E} N(E, t)]+Q(E, t)$

where $\dot{E}$ is the energy-loss rate of the particles with energy $E$. Energy-loss mechanisms include synchrotron radiation, inverse Compton scattering, and adiabatic losses.

The dynamics of the PWN in the supernova shell is calculated basically following the model presented in Gelfand et al. (2009). The model assumes that the progenitor supernova ejects material with mass $M_{\mathrm{ej}}$ and energy $E_{\mathrm{sn}}$ into the ambient matter with a constant density $\rho_{\mathrm{ISM}}$. Assuming the PWN has no influence on the dynamics of the forward shock and the reverse shock, the velocity and the radius of the forward shock and the reverse shock of the surrounding SNR are calculated with the equations in Truelove \& McKee (1999). The pulsar dissipates energy into the PWN, which sweeps up the supernova ejecta into a thin shell surrounding the nebula, and new particles are injected into the PWN at each time step.

We now apply the model to investigate the three composite SNRs, G0.9+0.1, MSH 15-52, and G338.3-0.0. First, if the distance, the age, and the radii of the SNR shell and the PWN are known, the parameters such as the supernova energy $\left(E_{\mathrm{sn}}\right)$, the ejecta mass $\left(M_{\mathrm{ej}}\right)$, the ambient density $\left(\rho_{\mathrm{ISM}}\right)$, and the initial spin-down energy $\left(\dot{E}_{0}\right)$ can be constrained by making the results consistent with the known values. In our calculation, a typical spin-down time scale $\left(\tau_{\mathrm{sd}}\right)$ is set to $500 \mathrm{yr}$ for the three remnants, and $\dot{E}_{0}$ is also constrained by the current spin-down power of the pulsar if it is available. Finally, to obtain a consistent nonthermal emmission with the multiband observational fluxes for the SNR, the other parameters can be constrained. Uncertainties for some parameters still exist owing to both their insensitivities in the final results and the uncertainties of the key properties of the system, such as the distance, the age, and the properties of the pulsar.

\section{1. $G 0.9+0.1$}

The composite SNR G0.9+0.1 consists of a bright 2' PWN hosted by a $8^{\prime}$ shell in the radio band (Helfand \& Becker 1987; La Rosa et al. 2000). A recent high-resolution radio study of the PWN indicates that the radio emission of the nebula has a spectral index of $-0.18 \pm 0.04$, and the fluxes are $1.35,1.45$, and $1.75 \mathrm{Jy}$ for the wavelengths $3.6,6$, and $20 \mathrm{~cm}$, respectively (Dubner et al. 2008). Furthermore, an energetic pulsar PSR J1747-2809 with a period of $52 \mathrm{~ms}$ has recently been discovered in G0.9+0.1 with the NRAO Green Bank Telescope at $2 \mathrm{GHz}$ (Camilo et al. 2009).

The PWN in G0.9+0.1 was detected in X-rays with BeppoSAX, and the result shows that the X-ray emission from 
Table 1. Input parameters for the three PWNe.

\begin{tabular}{lcccc}
\hline \hline Parameter & G0.9+0.1 & MSH 15-52 & G338.3-0.0 (case 1) & G338.3-0.0 (case 2) \\
\hline Distance $(\mathrm{kpc})$ & 8.5 & 5.2 & 10.0 & 10.0 \\
$T_{\text {age }}(\mathrm{yr})$ & 1900 & 1900 & 4500 & 8200 \\
$\tau_{\mathrm{sd}}(\mathrm{yr})$ & 500 & 500 & 500 & 500 \\
$M_{\mathrm{ej}}\left(M_{\odot}\right)$ & 8.0 & 8.0 & 8.0 & 8.0 \\
$E_{\mathrm{sn}}\left(10^{51} \mathrm{erg}\right)$ & 1.0 & 3.0 & 0.5 & 0.8 \\
$n_{\text {ism }}\left(\mathrm{cm}^{-3}\right)$ & 0.01 & 0.01 & 0.1 & 1.0 \\
$E_{0}\left(10^{39} \mathrm{erg} \mathrm{s}^{-1}\right)$ & 2.0 & 1.5 & 10 & 0.5 \\
$n$ & 3.0 & 2.84 & 3.0 & 3.0 \\
$\eta_{\mathrm{B}}\left(10^{-3}\right)$ & 1.0 & 20.0 & 1.0 & 0.3 \\
$\alpha$ & 2.5 & 2.4 & 2.5 & 2.5 \\
$E_{\mathrm{max}}(\mathrm{TeV})$ & 900 & 1000 & 500 & 500 \\
$E_{\mathrm{b}}\left(10^{5} \mathrm{MeV}\right)$ & 0.2 & 0.9 & 0.8 & 0.8 \\
\hline
\end{tabular}

the source has a power-law spectrum with a photon index of $2.0 \pm 0.3$ (Mereghetti et al. 1998; Sidoli et al. 2000). Highresolution observation with Chandra indicates that the nebula is axisymmetric with a jet-like feature, which is evidence of a torus of emission in the pulsar's equatorial plane and a jet directed along the pulsar spin axis (Gaensler et al. 2001). Proquet et al. (2003) studied G0.9+0.1 using observations by XMM-Newton. The X-ray spectrum softens with distance from the core, and the spectrum in the energy range $2-10 \mathrm{keV}$ has a power law form with a photon index of $\sim 1.9$.

Very high-energy (VHE) emission from G0.9+0.1 has been detected with HESS (Aharonian et al. 2005a). The photon flux above $0.2 \mathrm{TeV}$ is $5.7 \times 10^{-12} \mathrm{~cm}^{-2} \mathrm{~s}^{-1}$, and the spectrum can be fitted with a power law with a photon index $2.4 \pm 0.31$. The source is a weak TeV emitter, and the VHE $\gamma$-rays appear to originate in the core rather than the shell (Aharonian et al. 2005a).

The dynamical and radiative properties of the composite SNR G0.9+0.1 are investigated with the parameters in Table 1 for this source. Although the pulsar PSR J1747-2809 has a characteristic age of $5.3 \mathrm{kyr}$, Camilo et al. (2009) argue that G0.9+0.1 has a young age of no more than 2-3 kyr either from PWN evolution models of Blondin et al. (2001) for the observed ratio $R_{\mathrm{pwn}} / R_{\mathrm{snr}}=0.25$ or from the PWN energetics (Dubner et al. 2008). The distance of the pulsar is likely in the range of $8 \mathrm{kpc}$ to $16 \mathrm{kpc}$ due to the uncertainty of the electron density model toward the distant inner Galactic regions (Dubner et al. 2008). We assume the distance is $8.5 \mathrm{kpc}$ in the calculation, then the radii of the PWN and the shell are $2.55 \mathrm{pc}$ and $10.2 \mathrm{pc}$, respectively. Moreover, with $E_{\mathrm{sn}}=10^{51} \mathrm{erg}$ and $M_{\mathrm{ej}}=8 M_{\odot}$, an age of $1900 \mathrm{yr}$ and a relatively low density $n_{\text {ism }}=0.01 \mathrm{~cm}^{-3}$ are needed to reproduce the structure of the system correctly, i.e., the radius of the shell and the ratio $R_{\mathrm{pwn}} / R_{\mathrm{snr}}$. A pulsar's velocity of $120 \mathrm{~km} \mathrm{~s}^{-1}$ similar to the Crab pulsar (Kaplan et al. 2008 ) is used to illustrate the evolution of the PWN, which has no influence on the final results for G0.9+0.1 since it is a young remnant, and now the pulsar is safely inside the nebula. With these parameters, the resulting radii of the PWN and the SNR shell are 2.6 and $10.2 \mathrm{pc}$, respectively, consistent with the observational results (upper panel Fig. 1). The influence of the ejecta mass $M_{\mathrm{ej}}$ on the radius of the nebula is shown in the upper panel of Fig. 1. With a lower $M_{\mathrm{ej}}$, both the nebula and the SNR shell expand more quickly, and the nebula collides with the reverse shock at a later time. As a result, the magnetic field in the PWN is weaker for a lower $M_{\mathrm{ej}}$ because of the bigger volume of the nebula (lower panel Fig. 1).

The dynamical properties of the SNR during the evolution is similar to those for the Crab remnant in Gelfand et al. (2009)
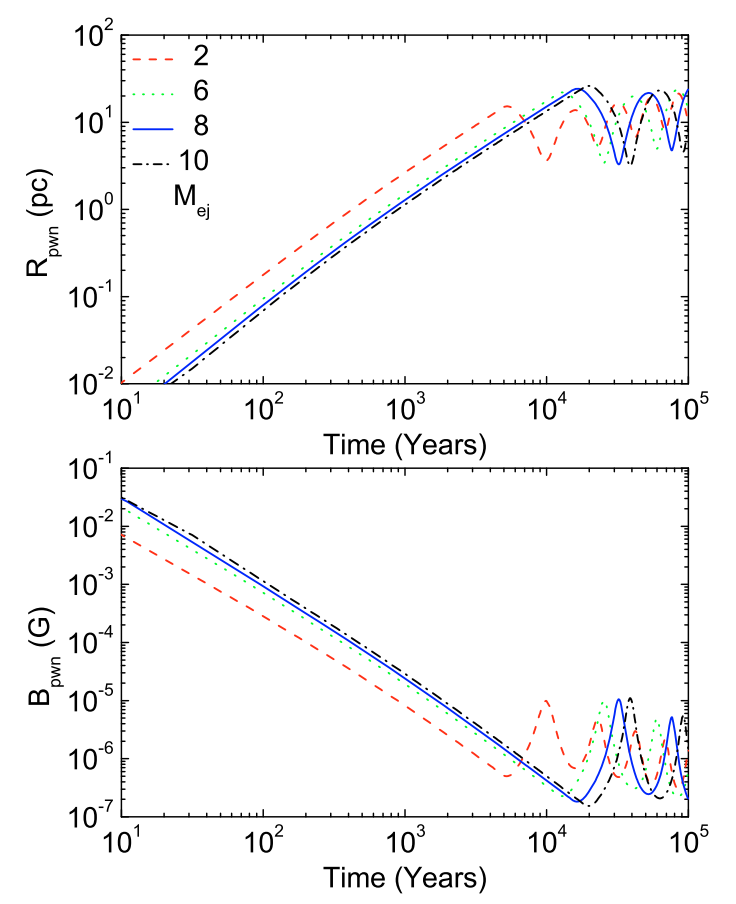

Fig. 1. Upper panel: radius of PWN $\left(R_{\mathrm{pwn}}\right)$ for $M_{\mathrm{ej}}=2$ (dashed line), $M_{\mathrm{ej}}=6$ (dotted line), $M_{\mathrm{ej}}=8$ (solid line) and $M_{\mathrm{ej}}=10$ (dash-dotted line) $M_{\odot}$ with the other parameters listed in Table 1 for G0.9+0.1. Lower right panel: magnetic field strength in the PWN $\left(B_{\text {pwn }}\right)$ with different $M_{\mathrm{ej}}$, and the others are same as the upper panel.

although the new spectrum of the particles is used in this paper. Initially, the pressure of the PWN is much greater than for the surrounding supernova ejecta, so the PWN expands adiabatically into the cold supernova ejecta. The ejecta surrounding the PWN is swept up to a thin shell, which is decelerated by ram pressure since its velocity is higher than the local sound speed (Gelfand et al. 2007). The mass of the PWN $M_{\text {pwn }}$ increases continuously since the shell expands faster than the ambient ejecta. This expansion phase ends when the PWN collides with the reverse shock of the SNR. After the collision, the pressure of the nebula $P_{\mathrm{pwn}}$ is much less than that of the supernova ejecta around the nebula $P_{\mathrm{snr}}\left(R_{\mathrm{pwn}}\right)$. The velocity of the PWN decreases a lot, and finally the PWN is compressed when the shell moves inward. During this process of compression, the magnetic field strength in the nebula increases significantly (lower panel in Fig. 1), and, as a result, the synchrotron luminosity has a rapid rise (lower right panel in Fig. 2). Furthermore, the radius of the 

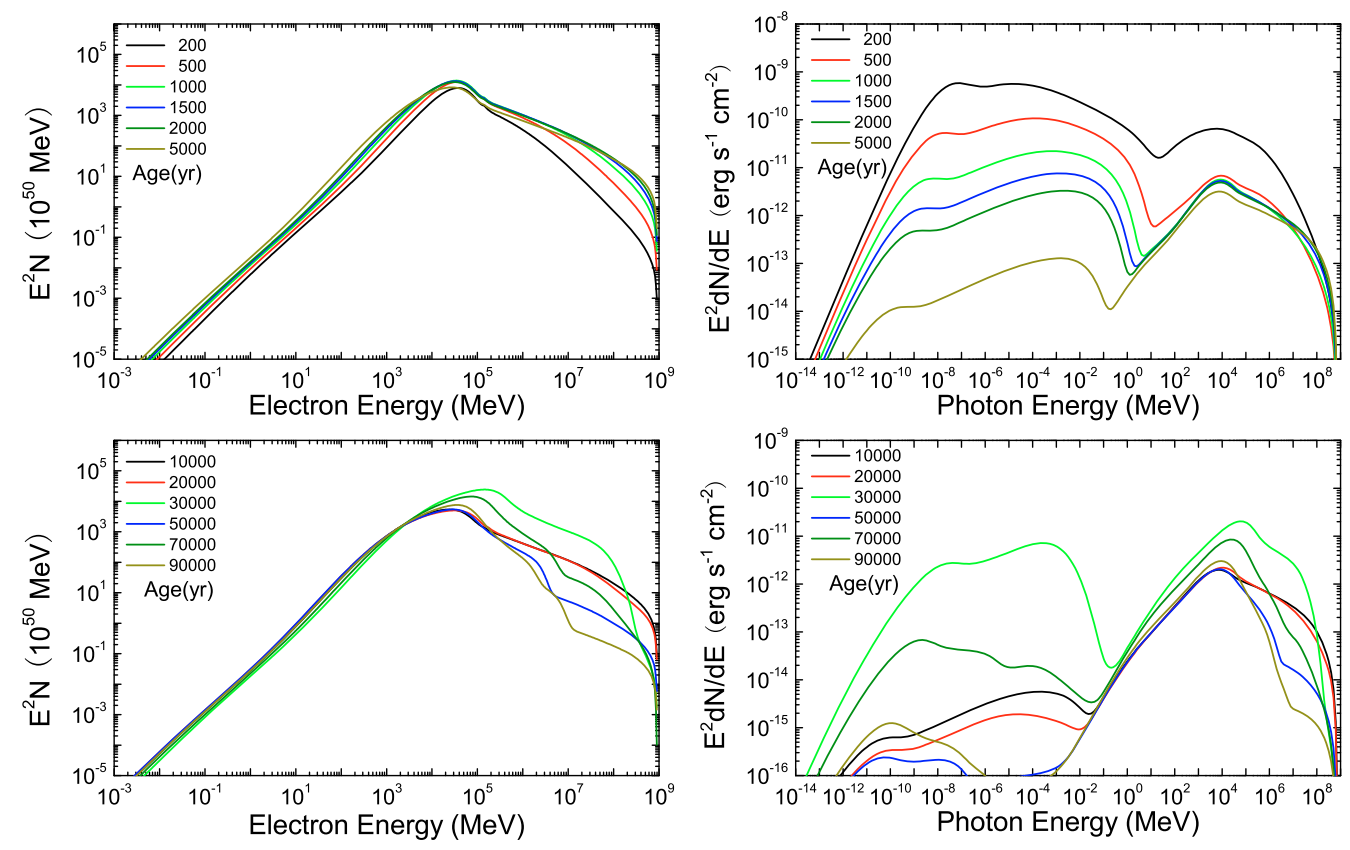

Fig. 2. Left panels: particle spectra of electrons and positrons at different times during the evolution of the PWN. Right panels: the resulting spectral energy distribution at different ages with the other parameters listed in Table.1.

PWN decreases significantly, and the PWN will expand again when the pressure in it eventually becomes greater than that of the surrounding ejecta. The nebula enjoys a series of contractions and re-expansions until the SNR enters the radiative phase of its evolution. The pulsar with a velocity moving in space will leave the PWN if the velocity is high enough in the compression process, and it can re-enter the nebula when the nebula expands again.

Particles with a spectrum of a Maxwellian plus a power-law tail ( $\alpha=2.5, f=1.0)$ are injected in the PWN when the pulsar is inside it. The particles lose energy through synchrotron radiation, inverse Compton scattering, and adiabatic loss. Nonthermal emission from the PWN from the radio to X-ray band is produced via synchrotron radiation, whereas $\gamma$-rays are produced through inverse Compton scattering on the seed photons, i.e., cosmic microwave background (CMB), infrared (IR), and optical (opt) photons. For the soft seed photons, the energy density of the infrared radiation is $0.23 \mathrm{eV} \mathrm{cm}^{-3}$ as used for the GALPROP code (Strong et al. 2000; Porter et al. 2006) and a density of $5.7 \mathrm{eV} \mathrm{cm}^{-3}$ for the optical component, which is $50 \%$ lower than the value used in GALPROP, are used in Aharonian et al. (2005a) to discuss the origins of the VHE $\gamma$-rays. We find densities of $0.5 \mathrm{eV} \mathrm{cm}^{-3}$ for the infrared component $\left(k T_{\mathrm{IR}}=3 \times 10^{-3} \mathrm{eV}\right)$, and $20 \mathrm{eV} \mathrm{cm}^{-3}$ for the optical soft photons $\left(k T_{\mathrm{opt}}=0.25 \mathrm{eV}\right)$ can reproduce the observed spectrum in the VHE $\gamma$-ray band, so these densities are used in this paper. The resulting energy distribution of the particles in the PWN and the multiwavelength, nonthermal emission during the evolution are presented in Fig. 2. The particles with energies below $E_{\mathrm{b}}$ are mainly from the highenergy particles with energies $>E_{\mathrm{b}}$ experienced energy losses, and the energy distributions in the range $<E_{\mathrm{b}}$ at different ages in the evolution all show a power-law form with an index of $\sim 1$ $\left(\mathrm{d} N / \mathrm{d} E \propto E^{-1}\right)$. On the other hand, the energy distribution in the higher-energy band ( $>E_{\mathrm{b}}$ ) becomes steady between $1000 \mathrm{yr}$ and $20000 \mathrm{yr}$, and the distribution can also be represented as a power law but with an index $\sim 2.5$.

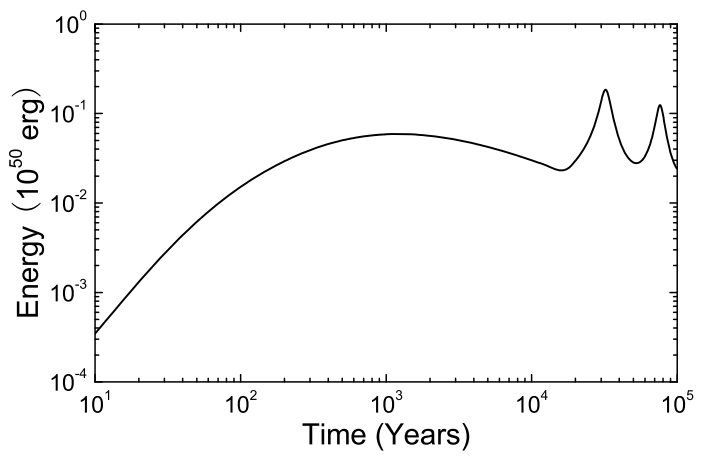

Fig. 3. The energy contained in the PWN at different ages of the system with the parameters same as in Fig. 2.

The expansion and compression of the PWN cause the magnetic field strength in the nebula $B_{\text {pwn }}$ to decrease and increase, respectively. The synchrotron emissivity diminishes before $10000 \mathrm{yr}$ as the PWN expands into the cold supernova ejecta, when the magnetic field strength in the nebula decreases gradually. However, synchrotron radiation can gain significance again when the PWN is compressed, because the magnetic field strength rises greatly during the compression. In this case, the energy of the particles in the nebula can show a rise caused by the adiabatic compression, then the emissivity of inverse Compton scattering can also show a rise (see the emission in Fig. 2 for $30000 \mathrm{yr}$ ). During the whole evolution process, the PWN is an important emitter of the $\mathrm{GeV} \gamma$-rays, although the emission from the radio to the $\mathrm{X}$-ray band is insignificant sometimes.

Figure 3 shows the evolution of the energy contained in the PWN $\left(E_{\mathrm{pwn}}\right)$ during the evolution of the system, and the spindown power of the pulsar and the adiabatic loss rate of the PWN, which can be obtained with $L_{\mathrm{ad}}=E_{\mathrm{pwn}} v_{\mathrm{pwn}} / R_{\mathrm{pwn}}$, are indicated in Fig. 4. Here $v_{\text {pwn }}$ and $R_{\text {pwn }}$ are the velocity and radius of the PWN. At first, the energy of PWN increases continuously since the pulsar injects the spin-down power into the nebula, and this stage ends after $\sim 1000 \mathrm{yr}$ (Fig. 3) when the adiabatic loss rate 
J. Fang and L. Zhang: Multiband emission from pulsar wind nebulae: a possible injection spectrum

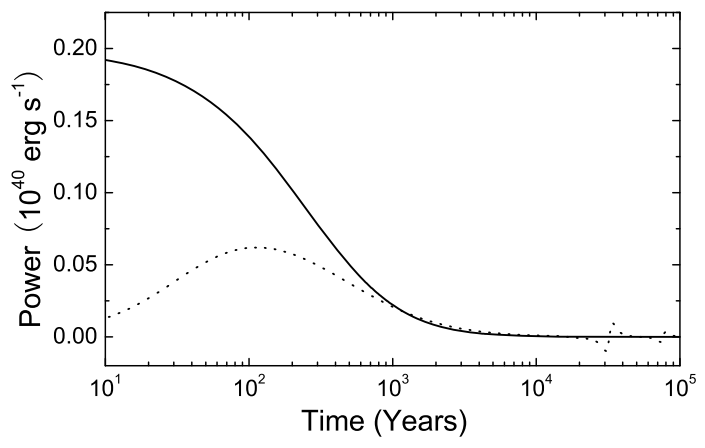

Fig. 4. The spin-down power of the pulsar (solid line) and the adiabatic loss rate of the PWN (dotted line) during the evolution of the system. The others are the same as in Fig. 3.

is considerably more than the spin-down power of the pulsar (Fig. 4). After this phase, the energy of the nebula decreases or increases because of the competition between the spin-down power of the pulsar adiabatic loss.

With the parameters in Table 1, the dynamical structure and the multiband radiative properties of $\mathrm{G} 0.9+0.1$ are reproduced at $1900 \mathrm{yr}$. At this age, the magnetic field strength in the PWN is $8.1 \mu \mathrm{G}$, and the current spin-down power of the pulsar is $8.67 \times 10^{37} \mathrm{erg} \mathrm{s}^{-1}$, which is still consistent with the measured one $4.3 \times 10^{37} \mathrm{erg} \mathrm{s}^{-1}$ (Camilo et al. 2009) thanks to the uncertainty of the moment of inertia; moreover, the observational radii of the nebula and the SNR shell are reproduced in this scenario. The resulting multiband emission of the PWN from the model is shown in the lower panel in Fig. 5. The radio and the X-ray observations can be well explained as synchrotron radiation of the particles injected in the PWN. The VHE $\gamma$-rays from the nebula are mainly produced by inverse Compton scattering on the optical light and the cosmic microwave background.

With a higher ejecta mass $\left(M_{\mathrm{ej}}\right)$, the radius of the PWN is smaller due to the deceleration of the ejecta matter before the collision between the reverse shock and the nebula. As a result, the resulting synchrotron radiation is stronger for a bigger $M_{\mathrm{ej}}$ (the upper panel in Fig. 5). Moreover, the influence of the break energy $E_{\mathrm{b}}$ on the resulting multiband nonthermal emission is indicated in Fig. 6 for $E_{\mathrm{b}}=0.1 \times 10^{5}, 0.2 \times 10^{5}, 0.5 \times 10^{5}$ and $1.0 \times 10^{5} \mathrm{MeV}$. With a higher $E_{\mathrm{b}}$, the resulting fluxes in $\mathrm{X}$-rays and $\gamma$-rays are higher. The multiband observational results in the radio, X-rays, and VHE $\gamma$-rays can be reproduced with $E_{\mathrm{b}}=0.2 \times 10^{5}$. In such a case, the Lorentz factor of the pulsar wind upstream of the TS can be estimated as $\sim 0.8 \times 10^{5}$.

\section{2. $M S H$ 15-52}

MSH 15-52 (G320.4-1.2) is a complex object located at a distance of $5.2 \pm 1.4 \mathrm{kpc}$ based on the HI absorption measurement, consistent with the value of $5.9 \pm 0.6 \mathrm{kpc}$ from the pulsar dispersion measure (Taylor \& Cordes 1993). It is a rough circular SNR with a diameter of $\sim 30^{\prime}$ from the radio observations (Caswell et al. 1981), and a PWN powered by an energetic pulsar PSR B1509-58 (e.g., Gaensler et al. 2002) was discovered in the remnant. Livingstone et al. (2005) presented an updated timing solution for the young energetic pulsar PSR B1509-58 based on the $21.3 \mathrm{yr}$ of radio data and the $7.6 \mathrm{yr}$ of X-ray timing data. The results show that the pulsar has a period of about $150 \mathrm{~ms}$ with a braking index of $n=2.839 \pm 0.003$, and a characteristic age of $\sim 1700 \mathrm{yr}$. In the radio band, the source appears as a shelllike SNR with bright spots in the NW and SE region (Mineo et al. 2001), and the NW region coincides with the IR and
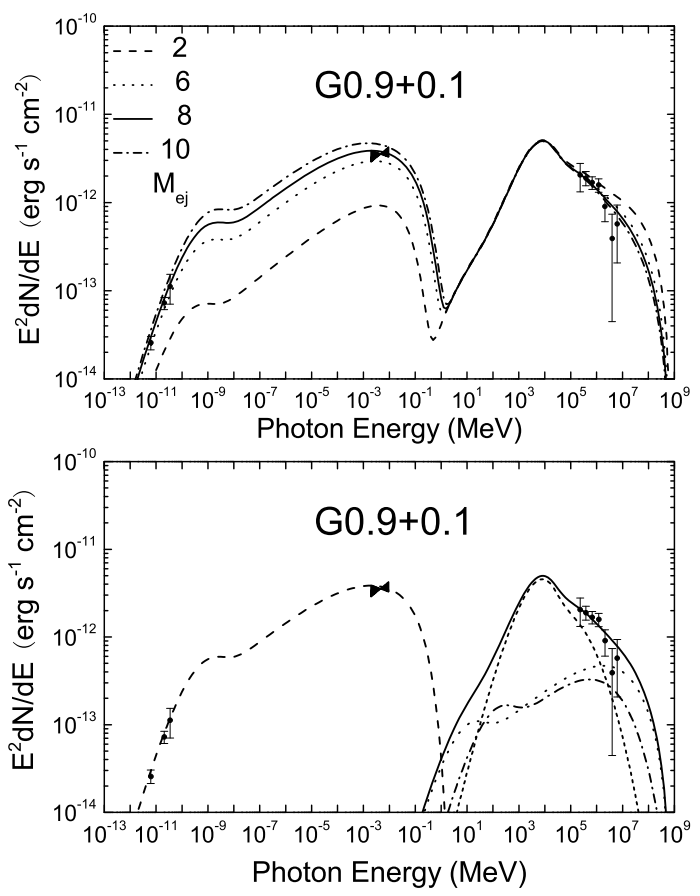

Fig. 5. Upper panel: the multiband spectral distribution of the nonthermal emission for $M_{\mathrm{ej}}=2$ (dashed line), $M_{\mathrm{ej}}=6$ (dotted line), $M_{\mathrm{ej}}=8$ (solid line), and $M_{\mathrm{ej}}=10$ (dash-dotted line) $M_{\odot}$ with the other parameters listed in Table 1 for G0.9+0.1. Lower panel: the resulting photon emission of synchrotron (dashed line), inverse Compton scattering on the CMB (dotted line), IR(dash-dotted line), starlight (short-dashed line), and synchrotron photons (close-dotted line) for $M_{\mathrm{ej}}=8 M_{\odot}$ and the others same as in the upper panel. The solid line represents the whole inverse Compton scattering of the electrons/positions on all the soft photons. The radio (Dubner et al. 2008), X-ray (Proquet et al. 2003), and VHE $\gamma$-ray (Aharonian et al. 2005a) observations for the PWN are also shown in the figure for comparison.

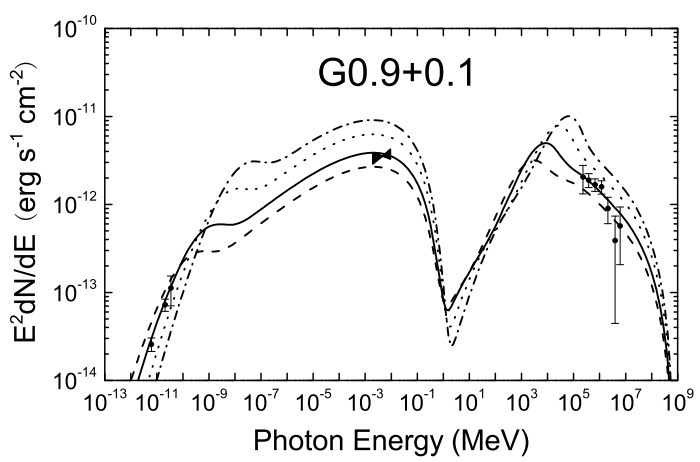

Fig. 6. The resulting spectral energy distributions for $E_{\mathrm{b}}=0.1 \times 10^{5}$ (dashed line), $0.2 \times 10^{5}$ (solid line), $0.5 \times 10^{5}$ (dotted line), and $1.0 \times 10^{5}$ (dash-dotted line) MeV. The others are the same as the lower panel in Fig. 5.

optical nebula RSW 89 (Seward et al. 1982). The PWN around the pulsar is comparatively faint in radio bands, and Gaensler et al. (2002) estimated a flux density of $2 \pm 1$ Jy at both 0.8 and $1.4 \mathrm{GHz}$ using the data of Whiteoak \& Green (1996) and Gaensler et al. (1999) for the PWN. In the X-ray band, diffuse emission is detected from the shell-like remnant with a bright spot coincident with the NW zone. The PWN has an elongated structure roughly centered on the pulsar with two arms extending several arcminutes along the NW and SE directions in the X-ray observations with ROSAT (Trussoni et al. 1996) and Chandra 
(Gaensler et al. 2002). The hard X-ray spectrum for the PWN was measured by BeppoSAX, and the power-law fit of the unpulsed high-energy flux gave a photon index $2.1 \pm 0.2$ and a flux of $1.2 \times 10^{-10} \mathrm{erg} \mathrm{cm}^{-2} \mathrm{~s}^{-1}$ in the $20-200 \mathrm{keV}$ energy range (Mineo et al. 2001). At VHE $\gamma$-ray energies, the SNR MSH 1552 has been observed with the HESS (Aharonian et al. 2005b) and CANGAROO (Nakamori et al. 2008). The TeV $\gamma$-rays are from an elliptically shaped region, and the jet extends more prominently to the south/southeast. This morphology coincides with the diffuse PWN as observed in the X-ray bands (Aharonian et al. 2005b). The overall energy spectrum can be fitted by a power law with photon index $\Gamma=2.27 \pm 0.03_{\text {stat }} \pm 0.02_{\text {syst }}$ in the energy range $280 \mathrm{GeV}$ to $40 \mathrm{TeV}$ in the HESS result, and a compatible result has been obtained with the CANGAROO telescope.

The age of the SNR MSH 15-52 has been estimated as 6-20 kyr with the standard parameters for the interstellar medium and for the supernova explosion (Seward et al. 1983), which is significantly older than the characteristic age (1700 yr) of pulsar. However, it is very likely that the system is young with an age of $\sim 1700 \mathrm{yr}$ since the SNR has expanded rapidly into a low-density cavity (Seward et al. 1983), which is supported by the observation of $\mathrm{HI}$ emission in this region (Dubner et al. 2002).

The resulting multiband nonthermal emission from $\mathrm{MSH}$ 15-52 is shown in Fig. 7, and the parameters are listed in Table1. With an initial spin-down power of $1.5 \times 10^{39} \mathrm{erg} \mathrm{s}^{-1}$ and $\tau_{0}=$ $500 \mathrm{yr}$, the spin-down power of the pulsar is $5.7 \times 10^{37} \mathrm{erg} \mathrm{s}^{-1}$ at an age of $1900 \mathrm{yr}$, consistent with the measured one, $5.4 \times$ $10^{37} \mathrm{erg} \mathrm{s}^{-1}$ (Livingstone et al. 2005). An energy density of $1.5 \mathrm{eV} \mathrm{cm}^{-3}$ for the optical soft photons is used in the calculation, which is consistent with the value from the GALPROP code (Strong et al. 2000; Porter et al. 2006). For the infrared component, we find a density of $1.5 \mathrm{eV} \mathrm{cm}^{-3}$ is needed to reproduce the observation in VHE $\gamma$-rays, so this value is employed in the calculation. With the parameters in Table1 for MSH 15-52, the radii of the PWN and the SNR shell are $3.6 \mathrm{pc}$ and $14.8 \mathrm{pc}$, respectively, and the resulting magnetic field strength inside the PWN is $19.3 \mu \mathrm{G}$ now. The observed emission from the radio (Gaensler et al. 2002) to the X-ray bands detected with BeppoSAX (Mineo et al. 2001) can be explained as the synchrotron radiation of the high-energy electrons/positrons injected in the nebula; moreover, the resulting emission from $100 \mathrm{MeV}$ to $10 \mathrm{TeV}$ is mainly produced via inverse Compton scattering off the soft infrared photons, and the HESS flux points can be reproduced fully.

\subsection{G338.3-0.0}

HESS J1640-465 was discovered in the survey of the inner Galaxy with HESS as a center-filled VHE $\gamma$-ray source with a differential energy spectrum fitted as a power law with an index of $2.42 \pm 0.14$ above $0.2 \mathrm{TeV}$ (Aharonian et al. 2006). This source is spatially coincident with the SNR G338.3-0.0, which has a broken shell with a diameter of $8^{\prime}$ indicated in the $843 \mathrm{MHz}$ radio survey using the Molonglo Observatory Synthesis Telescope (Whiteoak \& Green 1996). A highly absorbed source inside the shell was indicated in the X-ray observations with ASCA (Sugizaki et al. 2001) and Swift (Landi et al. 2006). Extended $\mathrm{X}$-ray emission centered on the VHE $\gamma$-rays was detected with a dedicated XMM-Newton observation in 2006 (Funk et al. 2007). Recently, Lemiere et al. (2009) present the high-resolution X-ray observations with Chandra on the extended source. The observed morphology shows a PWN and a point source presented as a potential pulsar. The spectrum of the putative pulsar and the

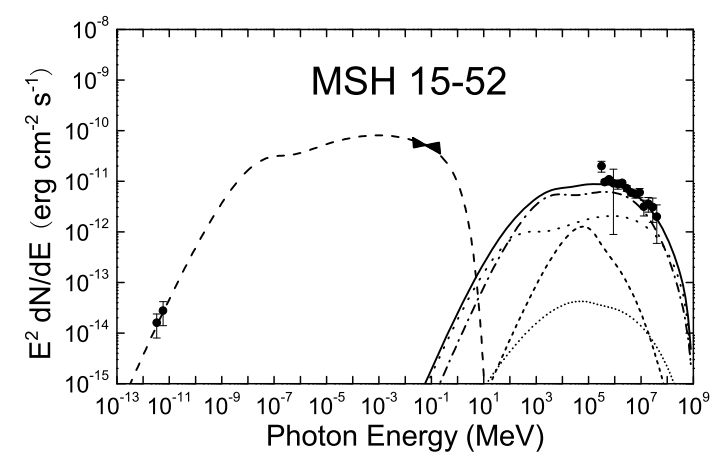

Fig. 7. Comparison of the resulting photon emission of synchrotron (dashed line), inverse Compton scattering on the CMB (dotted line), IR(dash-dotted line), starlight (short-dashed line), and synchrotron photons (close-dotted line) with the radio (Gaensler et al. 2002), X-ray (Mineo et al. 2001), and VHE $\gamma$-ray (Aharonian et al. 2005b) observations for the PWN in MSH 15-52. The parameters are listed in Table1 for this remnant.

PWN can be fitted with a power law with indices of 1.1 and 2.5, respectively (Lemiere et al. 2009). They argue that the pulsar's spin power is $\sim 4 \times 10^{36} \mathrm{erg} \mathrm{s}^{-1}$ based on the X-ray luminosities of the putative pulsar and nebula between 2 to $10 \mathrm{keV}$. The distance of the source is less certain, and it can be from $8 \mathrm{kpc}$ to $13 \mathrm{kpc}$ based on the $\mathrm{H} \mathrm{I}$ absorption features observed along the line of sight (Lemiere et al. 2009).

Assuming the SNR has a distance of $10 \mathrm{kpc}$, the radius of the radio shell is $\sim 12 \mathrm{pc}\left(4^{\prime}\right)$ (Whiteoak \& Green 1996), and that of the PWN is $\sim 3.5 \mathrm{pc}\left(1.2^{\prime}\right)$ in the X-rays according to the high-resolution observations with Chandra (Lemiere et al. 2009). Firstly, G338.3-0.0 is assumed to be a young SNR expanding into a tenuous medium with a density of $0.1 \mathrm{~cm}^{-3}$. The age of the remnant should not be too young since the PWN must contain enough energetic particles to produce the observational VHE $\gamma$-rays through inverse Compton scattering. As a result, the supernova explosion energy $E_{\mathrm{sn}}$ and the initial spin-down power of the pulsar are chosen to be $10^{51} \mathrm{erg}$ and $10^{40} \mathrm{erg} \mathrm{s}^{-1}$, respectively; moreover, $\mathrm{E}_{\max }$ is constrained to $500 \mathrm{TeV}$ to make the resulting emission consist with the observations in X-rays, and relatively high densities are needed to reproduce the VHE $\gamma$-ray fluxes detected with HESS, i.e., $5.0 \mathrm{eV} \mathrm{cm}^{-3}$ and $25.0 \mathrm{eV} \mathrm{cm}^{-3}$ for the infrared and the optical soft photons, respectively. At an age of $4500 \mathrm{yr}$, with the other parameters listed in Table1 for the SNR G338.3-0.0 (CASE 1), the radii of the shell, the reverse shock and the PWN are 11.1, 8.8 and 6.9 pc, respectively, and the magnetic field strength in the nebula is $3.7 \mu \mathrm{G}$. In such a case, the pulsar is energetic with a spin-down power of $1.0 \times 10^{38} \mathrm{erg} \mathrm{s}^{-1}$ now. Furthermore, the observed spectra in the X-rays with Chandra (Lemiere et al. 2009) and in the VHE $\gamma$-rays with HESS (Aharonian et al. 2006) can be reproduced (Fig. 8). Inverse Compton scattering on the optical light dominates the resulting emission at $0.1 \mathrm{TeV}$, whereas the emission above $1 \mathrm{TeV}$ is mainly produced via inverse Compton scattering off the IR photons.

On the other hand, we investigate the dynamical and radiative properties of G338.3-0.0 expanding into a medium with a density of $1 \mathrm{~cm}^{-3}$. With $E_{\mathrm{ej}}=0.8 \times 10^{51} \mathrm{erg}$, the radius of the shell of the SNR in radio means that the age of the remnant is $\sim 8000$ yr. With an initial spin-down power of $0.3 \times 10^{39} \mathrm{erg} \mathrm{s}^{-1}$ and other appropriate parameters (see Table1 for G338.3-0.0 CASE 2), the current spin-down power of the pulsar is $1.65 \times$ $10^{37} \mathrm{erg} \mathrm{s}^{-1}$, and the resulting radius of the PWN is just $2.45 \mathrm{pc}$, which is even smaller than the extension of the X-rays $(\sim 3.5 \mathrm{pc})$ 


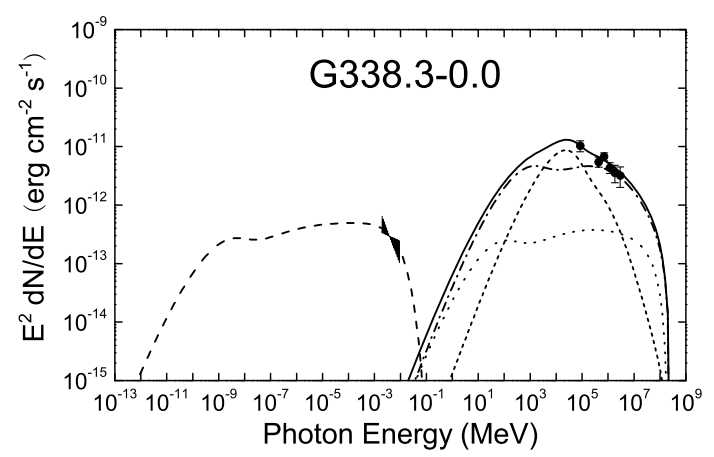

Fig. 8. Comparison of the resulting photon emission of synchrotron (dashed line), inverse Compton scattering on the CMB (dotted line), IR(dash-dotted line), starlight (short-dashed line), and synchrotron photons (close-dotted line) with the X-ray (Lemiere et al. 2009), and VHE $\gamma$-ray (Aharonian et al. 2006) observations for the PWN in G338.3-0.0. The parameters are listed in Table1 for G338.3-0.0 (CASE 1).

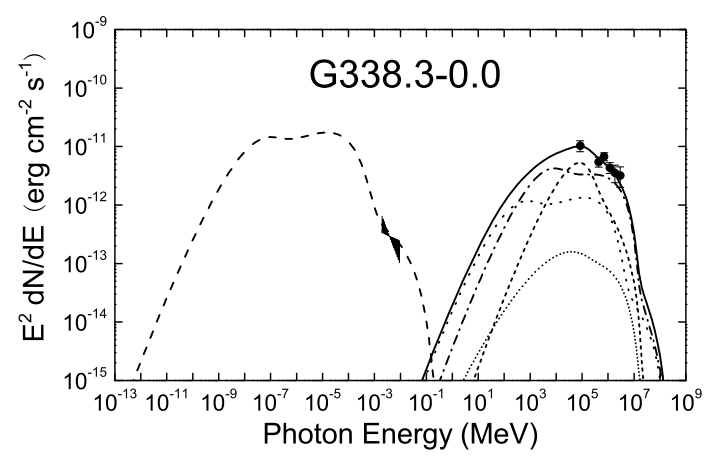

Fig. 9. The resulting spectral energy distribution with the parameters listed in Table1 for the CASE 2 of G338.3-0.0 (CASE 2). Others are the same as in Fig. 8.

assuming a distance of $10 \mathrm{kpc}$ (Lemiere et al. 2009). A smaller $\eta_{\mathrm{B}}=0.3 \times 10^{-3}$, and the maximum energy of the particles is set to $500 \mathrm{TeV}$ to reproduce the observational fluxes in the X-rays and $\gamma$-rays. The resulting multiband nonthermal emission is indicated in Fig. 9 with low densities of $1.0 \mathrm{eV} \mathrm{cm}^{-3}$ for the infrared and $6.0 \mathrm{eV} \mathrm{cm}^{-3}$ for the optical soft photons. In this scenario, the PWN has been compressed by the reverse shock, and the resulting flux with energies below $1 \mathrm{eV}$ is about two orders of magnitude higher than in the CASE 1.

\section{Discussion and conclusions}

Motivated by the finding that the spectrum of the particles downstream of a relativistic shock consists of two components: a relativistic Maxwellian and a power-law, high-energy tail with an index of $-2.4 \pm 0.1$ (Spitkovsky 2008), we investigate the possibility of particles with this new spectrum injected into PWNe from the TS based on the studies of multiband emission from PWNe. Following the dynamical method proposed in Gelfand et al. (2009), we studied the dynamical and multiband radiative properties of the three composite SNRs G0.9+0.1, MSH 15-52, and G338.3-0.0. With appropriate parameters, we find that a typical PWN is a strong $\gamma$-ray emitter during its evolution although the nonthermal radiation from the radio to the X-ray band is insignificant sometimes. The multiband observations of the three PWNe in the remnants can be reproduced with the new spectrum of the injected particles. Therefore, our studies of the dynamical and multiwavelength radiative properties of PWNe provide evidence that high-energy electrons/positrons can be injected into a PWN with a Maxwellian plus a power-law, high-energy tail from the TS of the PWN.

In modeling the multiband nonthermal emission from a PWN detected in the radio, X-ray, and $\gamma$-ray bands, particles injected with a spectrum of a broken power law are widely used to reproduce the observed multiwavelength emission (e.g., Venter \& de Jager 2006; Slane 2008; Zhang et al. 2008). Of course, for the three PWNe discussed in this paper, the multiband observed spectra of them can also be explained if the particles are injected with a broken power law. However, it is unclear why the broken power-law spectrum is valid when using it to reproduce the multiwavlength emission from a PWN. From our calculations, we have found that the energy distribution of the electrons/positrons in the nebula can be approximated as a broken power law with an index $\sim 1$ in the lower energy band and an index of $\sim 2.5$ in the higher-energy part before the PWN undergos significant compression, which is most likely the physical explanation of the broad use of a broken power law in modeling the multiband nonthermal emission from PWNe.

In this paper, high-energy electrons/positrons are injected into the PWN from the TS, and the main energy of the nebula is contained in these particles, which undergo radiative and adiabatic losses when the nebula evolves in the host SNR. Our study indicates that, for a typical PWN with the parameters similar as G0.9+0.1, the adiabatic loss of the particles in the nebula is significant after an age of $1000 \mathrm{yr}$ (see Figs. 3 and 4). Multiwaveband nonthermal emission from a PWN has been investigated using a simplified time-dependent injection model, in which high-energy electrons/positrons are injected into the PWN (e.g., Venter \& de Jager 2006; Slane 2008; Zhang et al. 2008). The pulsar inside the PWN transfers a part of its spindown power to the particles with a spectrum of a broken power law. In the simplified time-dependent injection model in Zhang et al. (2008), synchrotron loss of the particles is taken into account, whereas the adiabatic one is ignored. As a result, either a relatively weaker initial spin-down power of the pulsar or a lower efficiency of the power to the kinetic energy of the accelerated electrons/positrons is employed in the model. Moreover, in Zhang et al. (2008), an initial spin-down power of $1 \times 10^{38} \mathrm{erg} \mathrm{s}^{-1}$ for MSH 15-52 was used to investigate the multiband emission from the PWN, which is a factor of 15 less than used in this paper. Besides these reasons, another main one is the relatively large spin-down time scale of $\sim 5000 \mathrm{yr}$, which is $\propto \dot{E}_{0}^{-1}$ in the paper, used by Zhang et al. (2008), whereas in this paper it is adopted to be $500 \mathrm{yr}$. The energy released by the pulsar is mainly determined by $E_{0} \min \left\{T_{\text {age }}, \tau_{0}\right\}$, and the value in this paper is not much greater than in Zhang et al. (2008). Therefore, the multiband observed spectra for MSH 15-52 can be reproduced within the two scenarios even the initial spin-down power of the pulsar is significantly different.

Acknowledgements. We thank the referee, Dr. P. O. Slane, for his constructive comments. This work is partially supported by the Scientific Research Foundation of Graduate School of Yunnan University, the National Natural Science Foundation of China (NSFC 10778702, 10803005), a 973 Program (2009CB824800), and Yunnan Province under a grant 2009 OC.

\section{References}

Aharonian, F., Akhperjanian, A. G., Aye, K.-M., et al. (HESS Collaboration) 2005a, A\&A, 432, L25

Aharonian, F., Akhperjanian, A. G., Aye, K.-M., et al. (HESS Collaboration) 2005b, A\&A, 435, L17 
Aharonian, F., Akhperjanian, A. G., Bazer-Bachi, A. R., et al. (HESS Collaboration) 2006, ApJ, 636, 777

Atoyan, A. M., \& Aharonian, F. A. 1996, MNRAS, 278, 525

Blondin, J. M., Chevalier, R. A., \& Frierson, D. M. 2001, ApJ, 563, 806

Camilo, F., Ransom, S. M., Gaensler, B. M., \& Lorimer, D. R. ApJ, 700, L34

Caswell, J. L., Milne, D. K., \& Wellington, K. J. 1981, MNRAS, 195, 89

Dubner, G. M., Gaensler, B. M., Giacani, E. B., Goss, W. M., \& Green, A. J. 2002, AJ, 123, 337

Dubner, G., Giacani, E., \& Decourchelle, A. 2008, A\&A, 487, 1033

Funk, S., et al. 2007, ApJ, 267, 517

Gaensler, B. M., Brazier, K. T. S., Manchester, R. N., Johnston, S., \& Green, A. J. 1999, MNRAS, 305, 724

Gaensler, B. M., Pivovaroff, M. J., \& Garmire, G. P. 2001, ApJ, 556, L107

Gaensler, B. M., Arons, J., Kaspi, V. M., et al. 2002, ApJ, 569, 878

Gaensler, B. M., \& Slane, P. O. 2006, ARA\&A, 44, 17

Gelfand, J. D., Gaensler, B. M., Slane, P. O. et al. 2007, ApJ, 663, 468

Gelfand, J. D., Slane, P. O., \& Zhang, W. 2009, ApJ, 703, 2051

Goldreich, P., \& Julian, W. H. 1969, ApJ, 157, 869

Helfand, D. J., \& Becker, R. H. 1987, ApJ, 314, 203

Kaplan, D. L., Chatterjee, S., Gaensler, B. M., \& Anderson, J. 2008, ApJ, 677, 1201

La Rosa, T. N., Kassim, N. E., Lazio, T. J. W., \& Hyman, S. D. 2000, AJ, 119, 207

Landi, R., Bassani, L., Malizia, A., et al. 2006, ApJ, 651, 190

Lemiere, A., Slane, P., Gaensler, B. M., \& Murray, S. 2009, ApJ, 706, 1269

Livingstone, M. A., Kaspi, V. M., Gavril, F. P., \& Manchester, R. N. 2005, ApJ, 619, 1046

Mereghetti, S., Sidoli, L., \& Israel, G. L. 1998, A\&A, 331, L77
Mineo, T., Cusumano, G., Maccarone, M. C., et al. 2001, A\&A, 380, 695 Nakamori, T., Kubo, H.; Yoshida, T., et al. 2008, ApJ, 677, 297

Porquet, D., Decourchelle, A., \& Warwick, R. S. 2003, A\&A, 401, 197

Porter, T. A., Moskalenko, I. V., \& Strong, A. W. 2006, ApJ 648 , L29

Reynolds, S. P., \& Chevalier, R. A. 1984, ApJ, 278, 630

Seward, F. D., \& Harnden, F. R., Jr. 1982, ApJ, 256, L45

Seward, F. D., Harnden, F. R., Jr., Murdin, P., \& Clark, D. H. 1983, ApJ, 267, 698

Sidoli, L., Mereghetti, S., Israel, G. L., \& Bocchino, F. 2000, A\&A, 361, 719

Slane, P. 2008, AIPC, 1085, 120

Slane, P., Helfand, D. J., Reynolds, S. P., et al. 2008, ApJ, 676, L33

Spitkovsky, A. 2008, ApJ, 682, L5

Strong, A. W., Moskalenko, I. V., \& Reimer, O. 2000, ApJ, 537, 763

Sugizaki, M., Mitsuda, K., Kaneda, H., et al. 2001, ApJS, 134, 77

Taylor, J., H., \& Cordes, J. M. 1993, ApJ, 411, 674

Trussoni, E., Massaglia, S., Caucino, S., Brinkmann, W., \& Aschenbach, B. 1996, A\&A, 306, 581

Truelove, J. K., \& McKee, C. F. 1999, ApJS, 120, 299

van der Swaluw, E., Achterberg, A., Gallant, Y. A., \& Tóth, G. 2001, A\&A, 380, 309

van der Swaluw, E., Downes, T. P., \& Keegan, R. 2004, A\&A, 420, 937

Venter, C., \& de Jager, O. C. 2006, in Proc. 363rdWE-Heraeus Seminar, Neutron Stars and Pulsars, ed. W. Becker, \& H. H. Huang (MPE Report 291; Garching: MPE), 40

Volpi, D., Del Zanna, L., Amato, E., \& Bucciantini, N. 2008, A\&A, 485, 337

Whiteoak, J. B. Z., \& Green, A. J. 1996, A\&AS, 118, 329

Zhang, L., Chen, S. B., \& Fang, J. 2008, ApJ, 676, 1216 